\title{
Role of lumbar spine signal intensity measurement by MRI in the diagnosis of osteoporosis in post-menopausal women
}

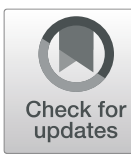

\author{
Maha M. Saad ${ }^{1}$, Ahmed Tohamy Ahmed ${ }^{1 *}$, Khaled E. Mohamed ${ }^{2}$ and Mohamed R. Habba ${ }^{1}$
}

\begin{abstract}
Background: Osteoporosis is a worldwide health problem and a common cause of bone fractures; the most common type of osteoporosis is post-menopausal type. MRI has a role in the diagnosis of osteoporosis and can be used as a screening tool, so the purpose of our study was to define a quantitative MRI-based score (M-score) for the detection of lumbar spine osteoporosis and to evaluate the correlation between lumbar spine signal intensity measured by MRI and BMD (bone mineral density) in post-menopausal women. Our case-control study involved 100 cases (50 old post-menopausal females as a case group and 50 healthy females as a control group of matched age). Both groups were subjected to history taking, dual-energy X-ray absorptiometry (DEXA), and conventional lumbar MRI. DEXA was performed for the lumbar spine and all scores (T-score, Z-score, BMD) were calculated. Lumbar MRI was performed (sagittal T1WI and T2WI) from L1-L4 levels. SNRL1-L4 and M-Score were calculated from T1W images.

Results: All DEXA scores were significantly lower in post-menopausal females compared to the control group $(P<$ 0.0001). Meanwhile, $S N R_{L 1-L 4}$ and M-score were significantly higher among cases than controls $(P<0.0001)$. The diagnostic threshold of SNR $\mathrm{L} 1-\mathrm{L} 4_{4}$ and M-score for distinguishing osteoporotic from non-osteoporotic females was 104.5 for $S N R_{L 1-L 4}$ with a sensitivity of $94 \%$, specificity $60 \%$, positive predictive values (PPV) $31 \%$, and negative predictive values (NPV) 98\%, and 3.5 for M-score with a sensitivity of $93.3 \%$, specificity $83.5 \%$, PPV $50 \%$, and NPV 98.6\%. SNR $\mathrm{L}_{\mathrm{L}-\mathrm{L} 4}$ was negatively correlated with M-score $(r=-0.74, P<0.0001)$, T-score $(r=-0.42, P<0.0001)$, Zscore $(r=-0.32, P<0.0001)$, and BMD $(r=-0.31, P<0.0001)$. M-score was negatively correlated with T-score $(r=-$ $0.48, P<0.0001)$, Z-score $(r=-0.36, P<0.0001)$, and BMD $(r=-0.37, P<0.0001)$. M-score moderately agreed with Tscore $(K=0.5, P<0.0001)$.

Conclusion: The MRI-based score (M-score) of the lumbar spine is an accurate quantitative method for distinguishing osteoporotic from non-osteoporotic females. M-score and $S N R_{L 1-L 4}$ are significantly correlated with osteoporotic indices (T-score and BMD).
\end{abstract}

Keywords: Osteoporosis, Lumbar spine, Magnetic resonance imaging, Dual-energy X-ray absorptiometry

\section{Background}

Osteoporosis is a worldwide health problem and is considered as the most common bone disease. It is proved that it is the main cause of bone fractures in elderly population. It is defined as a decreased mass per unit volume of normally mineralized bone $[1,2]$.

\footnotetext{
* Correspondence: Tohame_g@yahoo.com

'Department of Diagnostic Radiology, Faculty of Medicine, Suez Canal University, Ismailia, Egypt

Full list of author information is available at the end of the article
}

The most common type of osteoporosis is postmenopausal type (estrogen-deficient osteoporosis). About $15 \%$ of white people in their 50 s and $70 \%$ of those over 80 are affected [3]. Women are affected more than men [1]. In the developed world, depending on the method of diagnosis, $2-8 \%$ of males and $9-38 \%$ of females are affected $[4,5]$.

Dual-energy X-ray absorptiometry (DEXA) is considered as the gold standard for the diagnosis of osteoporosis as it uses negligible radiation dose and easy to use quantitative imaging modality that has high precision 
[6]. According to the World Health Organization guidelines, T-score helps to classify bone mineral density (BMD) [7]. Other methods rather than DEXA as quantitative computed tomography and quantitative ultrasound also have a role [8].

MRI has a role in detecting osteoporosis that the appearance of bone marrow is determined by its relative amount of protein, fat, water, and cells on MRI pulse sequence. The age-related changes of bone marrow revealed decreased cellularity and increased fat content, so this fact can be used as helpful information to detect the role of MRI in the diagnosis of osteoporosis in association with DEXA scan. T1-weighted spin-echo images are the best to evaluate the cellular content of the bone marrow [9].

So the purpose of our study is to assess the utility of quantitative MRI-based score (M-score) to detect osteoporosis in correlation with BMD measured by DEXA.

\section{Methods}

This is a case-control single-center study approved by the local institutional ethics committee; written informed consent was obtained from all patients.

\section{Study population}

Our case-control study was done over a period of time of 1 year from June 2016 to June 2017 and involved 50 patients and 50 normal volunteers who underwent lumbar MRI. Inclusion criteria included post-menopausal females $>50$ years who had undergone lumbar MRI for low back pain, and the reference control group of healthy females of matched age with normal body mass index (BMI $=19$ $25 \mathrm{~kg} / \mathrm{m}^{2}$ ) to calculate the MRI-based score equivalent to the T-score used in DEXA. Exclusion criteria were (a) MRI of the lumbar spine with contrast, (b) known patients with neoplastic disease, (c) traumatic dorso-lumbar vertebral injuries, (d) time elapsed between MRI examination and DEXA longer than 6 months, (e) absolute contraindication to MR imaging (claustrophobia, implanted defibrillator or pacemaker, cochlear implant, some types of clips used on brain aneurysms), and (f) patients refusing participation in the study.

All patients were subjected to history taking (age, menstrual, medical), DEXA scan, and conventional MRI assessment of the lumbar spine. Then the calculated quantitative MRI-based score (M-score) to detect osteoporosis was done and correlated with BMD measured by DEXA.

\section{DEXA scan}

DEXA scan for the lumbar spine was done for all participants by using DEXA GE model (DPx-pro). BMD, Zscore, and $\mathrm{T}$-score were calculated according to International Society for Clinical Densitometry (ISCD) guidelines (a minimum of two consecutive levels are used for lumbar T-score evaluation, after exclusion of vertebrae with focal structural abnormalities, degenerative changes, tubing artifact). The calculated values for the post-menopausal women ( $>50$ years) are compared with those of the healthy control group reference population values. T-scores are complicated statistical scores: $\mathrm{T}$ score $>1.0 \mathrm{SD}$ (normal), between -1.0 and $-2.5 \mathrm{SD}$ (osteopenia), $\leq-2.5 \mathrm{SD}$ (osteoporosis), and $\leq-2.5 \mathrm{SD}$ with 1 or more fragility fractures (severe osteoporosis).

\section{Magnetic resonance imaging methods and image analysis} All patients underwent lumbar MRI by using 1.5 Tesla MR scanner (Philips Medical Systems, Achieva) and post-processing was done by using a dedicated Philips workstation (Extended workspace, version 2.6).

\section{Patient's preparation}

The ferromagnetic materials were taken off. The procedure was explained to the patient, and then the patient was asked to lie supine and instructed not to move during the study.

\section{MRI protocol and technique}

Routine lumbar spine assessment from L1-L4 was performed usually by obtaining sagittal T1W spin-echo sequence $(\mathrm{TR}=7, \mathrm{TE}=400-600$, slice thickness $=4 \mathrm{~mm}$, fov $=280 \mathrm{~mm}$, matrix $=320 \times 320$ ), the most useful sequence for the assessment of bone marrow. Region of interest (ROI) was manually placed as a circle in the vertebral body excluding the cortical bone, subchondral abnormalities, focal lesions (e.g., hemangioma), and posterior venous plexus. Three ROIs were used for each vertebra, and each of them acquired on a different slice (with their mean used for analysis; that signal-to-noise ratio is not equivalent within vertebrae). Another ROI was placed in an artifact-free site outside the patient to measure the noise.

\section{MRI image analysis}

All MR images were reviewed by a consensus of two experienced radiologists blinded to the clinical information and DEXA scan results. Images were transferred to an offline workstation for post-processing. Signal-to-noise ratio (SNR) was obtained by dividing the intra-vertebral intensity by the standard deviation of the noise.

The diagnostic performance of $\mathrm{SNR}_{\mathrm{L} 1-\mathrm{L} 4}$ was estimated for each patient and used to obtain M-score (MRI-based score) for the diagnosis of osteoporosis. The $\mathrm{SNR}_{\mathrm{L} 1-\mathrm{L} 4}$ of the control group was also used in the equation by using their mean $\left(\mathrm{SNR}_{\mathrm{ref}}\right)$ and standard deviation $\left(\mathrm{SD}_{\mathrm{ref}}\right)$ [10].

The M-score was defined according to the formula as follows:

$$
\mathrm{M} \text {-score }=\frac{\mathrm{SNR}_{\mathrm{L} 1-\mathrm{L} 4}-\mathrm{SNR}_{\mathrm{ref}}}{\mathrm{SD}_{\mathrm{ref}}}
$$




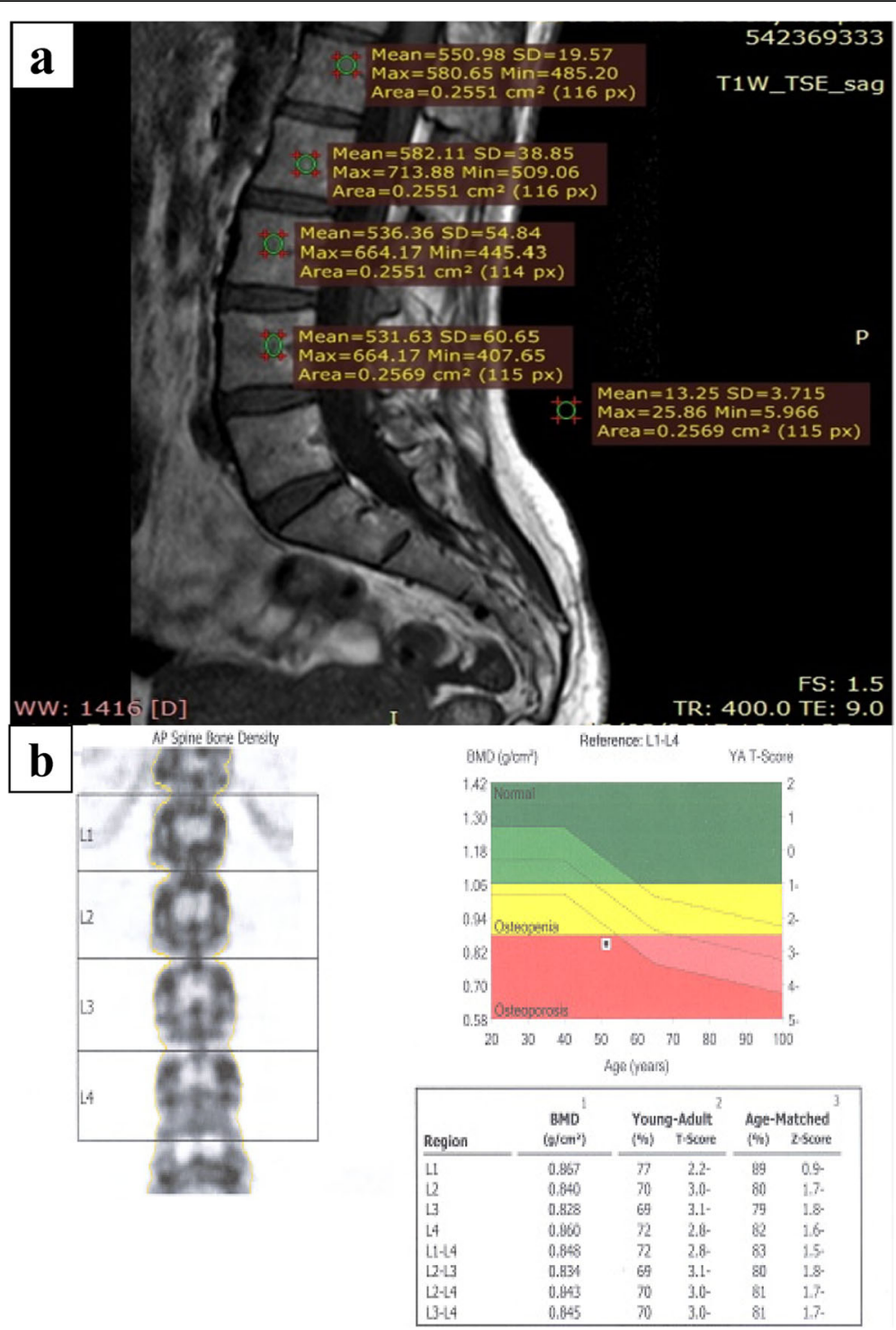

Fig. 1 Lumbar MRI of a 55-year-old post-menopausal woman complained from low back pain, no history of any chronic illness, spinal trauma, family history of osteoporosis, history of taking any medications, or taking calcium and Vit. D supplementation. MRI of lumbar spine T1W (L1-L4) revealed that the $S N R_{L 1-L 4}=148.5$ and $M-s c o r e$ within abnormal values $=4.1093$, DEXA scan of the lumbar spine, PA (L1-L4) revealed osteoporosis according to T-score results. The mean BMD of the lumbar spine is $0.847 \mathrm{~g} / \mathrm{cm}^{2}$, equivalent to a T score of -2.8

\section{Statistical analysis}

Statistical analysis and tests were specified according to the variable type. A commercially available PC-based software package (SPSS) was used.

Results were compared with the final diagnosis and the predictive values of MRI sequences were calculated by obtaining positive predictive values (PPV), negative predictive values (NPV), sensitivity, specificity, and total accuracy in the detection of osteoporosis.

Receiver operating curve (ROC) analysis was used to define the cutoff values of $\mathrm{M}$-score and $\mathrm{SNR}_{\mathrm{L} 1-\mathrm{L} 4}$ for the discrimination of osteoporotic from nonosteoporotic females.
Chi-squared test was used for analyzing differences between the results of MRI (calculated M-score) and other imaging modalities (DEXA scan). The sensitivity and specificity of MRI and other imaging modalities were calculated and compared.

\section{Results}

The study involved 50 post-menopausal women with a mean age of $59.4+4.1$ years (age range $46-68$ years) and 50 healthy females of matched ages. BMI was significantly higher among post-menopausal women in comparison to healthy controls $\left(33.8 \mathrm{~kg} / \mathrm{m}^{2}\right.$ versus $22.01 \mathrm{~kg} /$ $\mathrm{m}^{2}$, respectively; $P$ value $\left.<0.0001\right)$. 


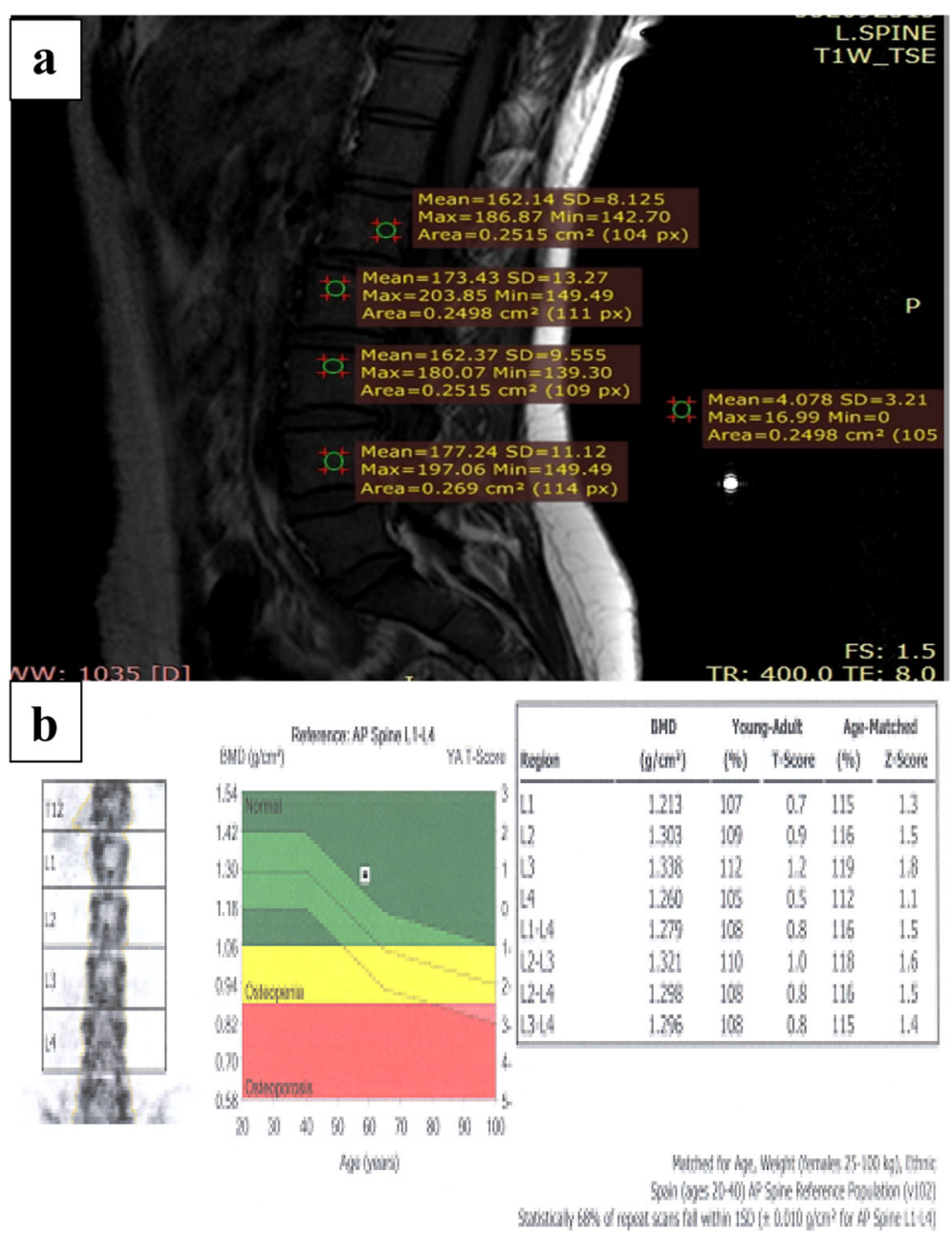

Fig. 2 Lumbar MRI of a 58-year-old post-menopausal woman with a history of low back pain. No history of any chronic illness, spinal trauma, family history of osteoporosis, history of taking any medications, or taking calcium and vit. D supplementation. MRI of lumbar spine T1W (L1-L4) revealed that the $S N R_{L 1-L 4}=53.93$, and M-score $=0.045$ within normal values, DEXA scan of the lumbar spine, and PA (L1-L4) revealed normal Tscore results. The mean BMD of the lumbar spine is $1.27 \mathrm{~g} / \mathrm{cm}^{2}$, equivalent to a T-score of 0.8

All DEXA scores including T-score, Z-score, and BMD were significantly lower in post-menopausal females compared to the control group $(P$ value $<0.0001)$. Meanwhile, $\mathrm{SNR}_{\mathrm{L} 1-\mathrm{L} 4}$ and $\mathrm{M}$-score were significantly higher among cases than controls $(P$ value $<0.0001)$ (Figs. 1 and 2). According to WHO criteria, of the case group, it was evoked that 11 patients $(22 \%)$ had a normal T-score, 24 patients (48\%) had osteopenia, and 15 patients (30\%) had osteoporosis. Obesity was representing the most co-morbid condition related to osteoporosis that was present in the majority of patients $(82 \%)$.

The ROC curve assessment of $\mathrm{SNR}_{\mathrm{L} 1-\mathrm{L} 4}$ for the discrimination of osteoporotic from non-osteoporotic females revealed that $\mathrm{AUC}=0.696$ at $95 \%$ confidence interval $0.636-$ 0.981 and the diagnostic capability of using a threshold of 104.5 as a cutoff had a sensitivity of $94 \%$, specificity $60 \%$, PPV 30.6\%, and NPV 98\% ( $P$ value $=0.0043$; Fig. 3 ).
The ROC curve assessment of M-score for the discrimination of osteoporotic from non-osteoporotic females revealed that $\mathrm{AUC}=0.904$ at $95 \%$ confidence interval $0.636-0.981$ and the diagnostic capability of using a threshold of 3.5 as a cutoff had a sensitivity of 93.3\%, specificity $83.5 \%$, PPV 50\%, and NPV 98.6\%; $P$ value $<0.0001$ (Fig. 4). Meanwhile, the ROC curve assessment of $\mathrm{M}$-score for the discrimination of postmenopausal from healthy control females revealed that AUC $=0.975$ at $95 \%$ confidence interval $0.636-0.981$ and the diagnostic capability of using a threshold of 2.2 as a cutoff had a sensitivity of $92 \%$, specificity 98\%, PPV 97.9\%, and NPV 92.5\% ( $P$ value $<0.0001$; Fig. 5$)$.

The study showed that $\mathrm{SNR}_{\mathrm{L} 1-\mathrm{L} 4}$ was negatively correlated with $\mathrm{M}$-score $(r=-0.74, P$ value $<0.0001)$, T-score $(r=-0.42, P$ value $<0.0001)$, Z-score $(r=-0.32, P$ value $<$ $0.0001)$, and BMD $(r=-0.31, P$ value $<0.0001)$ (Table 1$)$. 


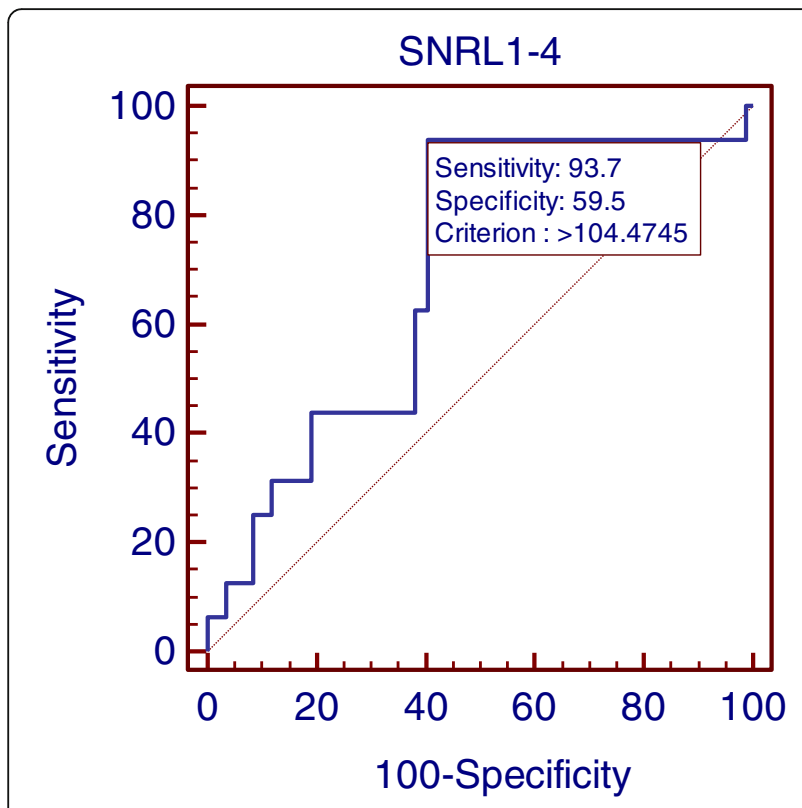

Fig. 3 Receiver operating characteristic (ROC) curve for SNR $R_{L 1-L 4}$ for the discrimination of osteoporotic from non-osteoporotic females revealed that by using a threshold of 104.5 as a cutoff value had a sensitivity of 94\%, specificity 60\%, PPV 30.6\%, and NPV 98\% at a $P$ value $=0.0043$

The ROC curve assessment of M-score for the discrimination of normal from low BMD revealed that AUC $=0.696$ at $95 \%$ confidence interval $0.636-0.981$ and the diagnostic capability of using a threshold of 2.8 as a cutoff had a sensitivity of $53.8 \%$, specificity $88.6 \%$, PPV $89.7 \%$, and NPV 50.8\% ( $P$ value $=0.0007 ;$ Fig. 6$)$.

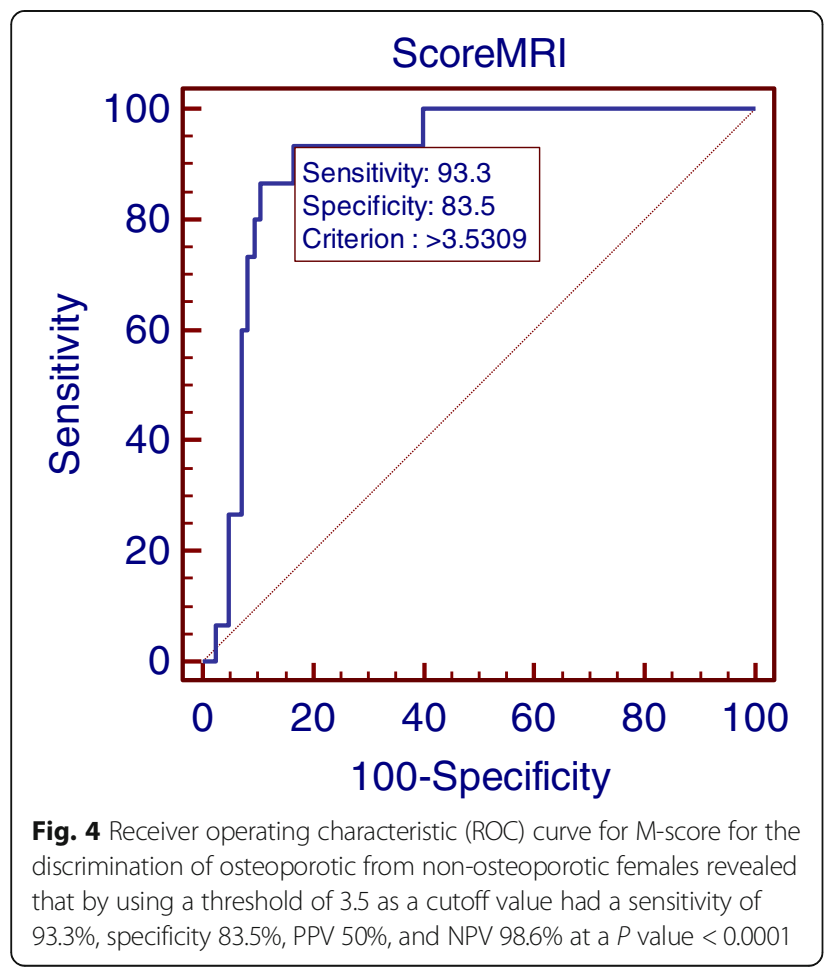

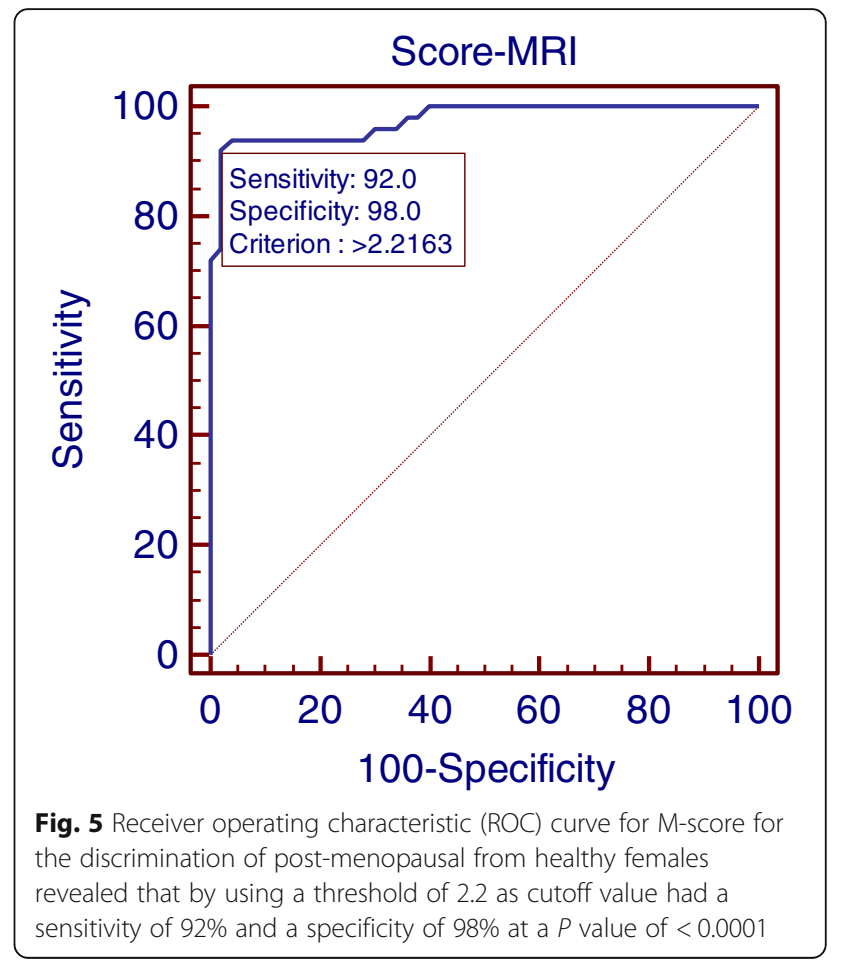

The study revealed that M-score was negatively correlated with $\mathrm{SNR}_{\mathrm{L} 1-\mathrm{L} 4}(r=-0.74, P$ value $<0.0001)$, Tscore $(r=-0.48, P$ value $<0.0001)$, Z-score $(r=-0.36, P$ value $<0.0001)$, and BMD $(r=-0.37, P$ value $<0.0001)$ (Table 2).

\section{Discussion}

Several studies had used CT modality for evaluating osteoporosis and had been performed in the last decade which demonstrated how routine abdominal CT obtained for other clinical indications could be used for quantitative diagnosis of osteoporosis [11], and also, demonstrated the possibility of accurately detecting osteoporosis-related fragility fractures by CT [12, 13]. As there are more than 80 million CT examinations performed each year in the USA, the idea of extracting

Table 1 Correlations between signal-to-noise ratio (SNR) $L$ 1-L4 and radiological indices using magnetic resonance imaging (MRI) and dual-energy X-ray absorptiometry (DEXA)

\begin{tabular}{lll}
\hline & \multicolumn{1}{c}{ SNR $R_{L 1-L 4}$} & \\
\cline { 2 - 3 } & Pearson's correlation coefficient $(r)$ & ${ }^{*} P$ value (two-tailed) \\
\hline M-score & -0.743 & $<0.0001^{* *}$ \\
T-score & -0.418 & $<0.0001^{* *}$ \\
Z-score & -0.315 & $0.001^{* *}$ \\
BMD & -0.312 & $0.002^{* *}$ \\
\hline
\end{tabular}

${ }^{*}$ Correlation was significant at p-value $<0.05$

${ }^{* *}$ Correlation was highly significant at $P$ value $<0.01$ 


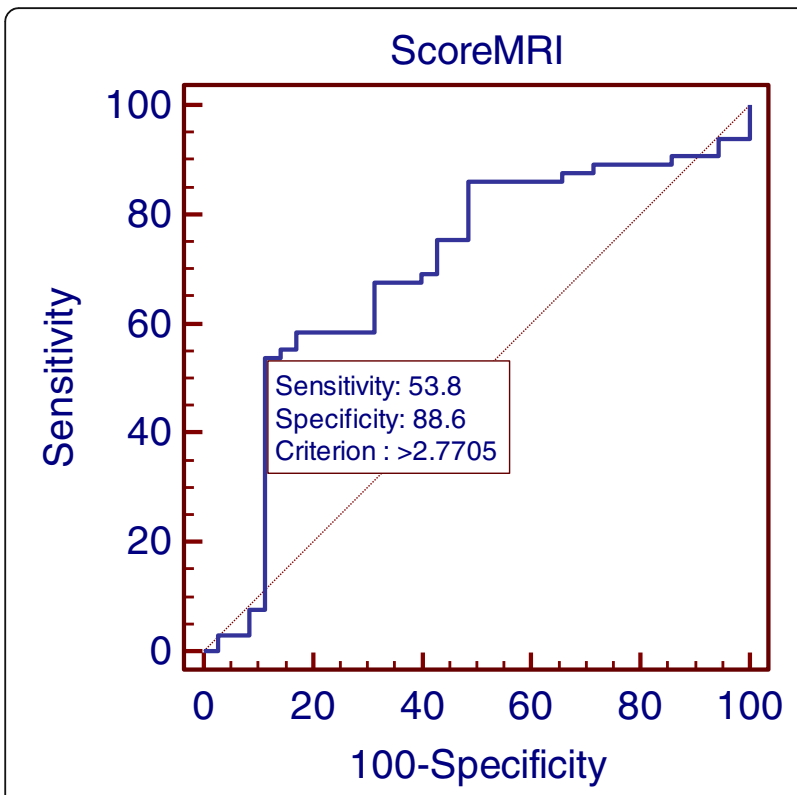

Fig. 6 Receiver operating characteristic (ROC) curve for M-score for the discrimination of normal from low BMD revealed that by using a threshold of 2.8 as a cutoff value had a sensitivity of $53.8 \%$ and a specificity of $88.6 \%$ at a $P$ value $=0.0007$

more information from imaging data collected for other purposes holds merit [14].

Regarding MRI, previous studies demonstrated that the bone marrow signal intensity on the standard T1weighted images is negatively correlated with BMD and osteoporosis as shown by Shen et al.$[15,16]$ and Shah and Hanrahan [9]. This correlation may open a window for the possibility to detect osteoporosis using MRI. Moreover, osteoporosis-related fragility fractures may be accurately detected by MRI as shown by Bazzocchi et al. [12]. Only one study by Bandirali et al. [10] had previously aimed to define a new quantitative lumbar spine MRI-based method on standard T1-weighted images for diagnosing osteoporosis.

The MRI-based score (M-score) can be calculated easily that lumbar spine MRI is the routine and commonest examination for low back pain assessment. From the aspect of being a common examination for low back pain,

Table 2 Correlations between M-score and radiological indices using magnetic resonance imaging (MRI) and dual-energy X-ray absorptiometry (DEXA)

\begin{tabular}{lll}
\hline & M-score & \\
\cline { 2 - 3 } & Pearson's correlation coefficient $(r)$ & ${ }^{*} P$ value (two-tailed) \\
\hline SNR $_{\mathrm{L} 1-\mathrm{L} 4}$ & -0.743 & $<0.0001^{* *}$ \\
T-score & -0.484 & $<0.0001^{* *}$ \\
Z-score & -0.356 & $<0.0001^{* *}$ \\
BMD & -0.368 & $<0.0001^{* *}$ \\
\hline
\end{tabular}

*Correlation was significant at $P$ value $<0.05$

${ }^{* *}$ Correlation was highly significant at $P$ value $<0.0001$ so it can be used as a screening tool for the detection or suspicion of osteoporosis in patients.

We agreed with a study done by Bandirali et al. [10] in the main concept and high sensitivity and specificity of M-score with its high utility as a screening tool. Our study revealed sensitivity and specificity values around $93 \%$ and $84 \%$, respectively, of M-score correlated with DEXA examination as a reference gold standard. Based on our sample which involved 100 subjects, it was found that $\mathrm{SNR}_{\mathrm{L} 1-\mathrm{L} 4}$ was negatively correlated with $\mathrm{BMD}(r=$ $-0.3, P<0.0001)$, compared to $(r=-0.67, P<0.001)$ in Bandirali et al.'s study.

It was found in our study that the threshold of Mscore was 3.5 with a sensitivity of $93.3 \%$, specificity 83.5\%, PPV 50\%, and NPV 98.6\%, and this means that for every 100 patients with $\mathrm{M}$-score $\geq 3.5$, 93 patients will have a true positive rate or really identified as to have osteoporosis. This also means that for every 100 patients with M-score < 3.5, 84 subjects will have a true negative rate or correctly identified as not having osteoporosis. In Bandirali et al.'s study, the demonstrated threshold of M-score by them was 5.5 with a sensitivity of $54 \%$. The difference in the threshold of M-score could be due to the sample size and sample type, as our sample intently included post-menopausal women which are at high risk of development of osteopenia or osteoporosis due to hormonal changes and obesity as a common co-morbid finding as described below. In obese patients and patients with spinal degenerative disease, lumbar BMD may be increased and gives the false impression that these patients have a low risk of fractures $[17,18]$.

But with DEXA scan, the diagnosis of osteoporosis depends on T-score and not BMD which gives more easy assessment away from calibration difficulties in the case of using BMD [6]. So M-score measured by MRI was used as a predictor of osteoporosis and a parameter to be correlated with T-score calculated by DEXA with good diagnostic performance.

Our study has several strengths that may be deserved to be mentioned. First, MRI and DEXA were simultaneously performed in two-thirds of the patients (66\%). In the rest of the patients (34\%), the median time elapsed between the two examinations was low (about 33 days) and it is unlikely to have influenced the correlation between DEXA and MRI.

Second, regarding the reference group of a healthy woman undergoing MRI, bone densitometry data were also available because they undergo DEXA as well. Moreover, we excluded the healthy females with any abnormalities considering that they must have healthy bone status.

Third, we excluded post-menopausal women with vertebral osteoporotic fractures. That on DEXA, patients with low-trauma fractures have non-reliable non-osteoporotic BMD. 
This study had also some limitations; firstly, the use of $\mathrm{M}$-score threshold values will be different for each single-center according to different SNRs values provided by each MRI system. Secondly, the signal-to-noise ratio assessment by the placement of the region of interest (ROI) placed within the vertebral body excluding the cortical bone, subchondral abnormalities, focal lesions (e.g., hemangiomas), and posterior venous plexus and outside of the patient are simple methods of measurement as compared to spectroscopic measurement $[19,20]$. This study is specific for post-menopausal women; however, other multicenter studies are mandatory for both males and females as also males $>50$ years have a higher risk for osteoporosis.

\section{Conclusion}

In conclusion, we recommended to include the measurement of $\mathrm{M}$-score and $\mathrm{SNR}_{\mathrm{L} 1-\mathrm{L} 4}$ as part of the routine evaluation of the lumbar spine, that it is considered an easy, reliable, and reproducible screening tool for osteoporosis without any additional imaging, radiation exposure, especially in post-menopausal women or patients at risk for osteoporosis that will help to early detect persons who need medical advice, prevent the disease and improve patient's outcome.

\section{Abbreviations}

BMD: Bone mineral density; DEXA: Dual-energy X-ray absorptiometry

\section{Acknowledgements}

Not applicable

\section{Ethical approval and consent to participate}

Approved by the local institutional ethics committee (Committee of Scientific Research Ethics (CSRE), Suez Canal University, Egypt); written informed consent was obtained from all patients.

\section{Availability of data and materials}

The dataset used and/or analyzed during the current study are available from the corresponding author on reasonable request.

\section{Authors contributions}

MM helped in the preparation of the study methodology, data collection, and analysis of data. AT participated in the formulation of the study, preparation of methodology, data collection, analysis and revision of the collected data, and writing the paper. KE participated in the supervision of the study methodology and collected the data. MR participated in the revision of the study methodology and collected the data. All authors have read and approved the final manuscript.

\section{Funding}

The authors state that this work has not received any funding.

\section{Consent for publication}

Consent for publication was obtained from the patients.

\section{Competing interests}

The authors declare that they have no competing interests.
Received: 26 June 2019 Accepted: 6 September 2019

Published online: 18 September 2019

\section{References}

1. Colón-Emeric CS, Saag KG (2006) Osteoporotic fractures in older adults. Best Pract Res Clin Rheumatol 20:695-706. https://doi.org/10.1016/j.berh. 2006.04.004

2. Golob AL, Laya MB (2015) Osteoporosis: screening, prevention, and management. Med Clin North Am 99:587-606. https://doi.org/10.1016/j. mcna.2015.01.010

3. WHO. "Chronic rheumatic conditions". World Health Organization. 2015; Retrieved 18 May.

4. Wade SW, Strader C, Fitzpatrick LA et al (2014) Estimating prevalence of osteoporosis: examples from industrialized countries. Arch Osteoporos:182. https://doi.org/10.1007/s11657-014-0182-3,9

5. Willson T, Nelson SD, Newbold J et al (2015) The clinical epidemiology of male osteoporosis: a review of the recent literature. Clin Epidemiol 7:65-76. https://doi.org/10.2147/CLEP.S40966

6. Bandirali M, Lanza E, Messina C et al (2013) Dose absorption in lumbar and femoral dual energy $\mathrm{X}$-ray absorptiometry examinations using three different scan modalities: an anthropomorphic phantom study. J Clin Densitom 16:279-282. https://doi.org/10.1016/j.jocd.2013.02.005

7. WHO (1994) World Health Organization Technical report: assessment of fracture risk and its application to screening for postmenopausal osteoporosis: a report of WHO study group. Switzerland, Geneva

8. Link TM (2012) Osteoporosis imaging: state of the art and advanced imaging. Radiology 263:3-17. https://doi.org/10.1148/radiol.2633201203

9. Shah LM, Hanrahan CJ (2011) MRI of spinal bone marrow: part I, techniques and normal age-related appearances. AJR Am J Roentgenol 197:1298-1308. https://doi.org/10.2214/AJR.11.7005

10. Bandirali M, Di LeG, PapiniG, et al (2015) A new diagnostic score to detect osteoporosis in patients undergoing lumbar spine MRI. Eur Radiol 25:2951-2959.

11. Pickhardt P, Pooler B, Lauder T et al (2013) Opportunistic screening for osteoporosis using abdominal computed tomography scans obtained for other indications. Ann Intern Med 158:588-595

12. Bazzocchi A, Garzillo G, Fuzzi F et al (2014) Localizer sequences of magnetic resonance imaging accurately identify osteoporotic vertebral fractures. Bone 61:158-163

13. Bazzocchi A, Spinnato P, Albisinni $U$ et al (2012) A careful evaluation of scout CT lateral radiograph may prevent unreported vertebral fractures. Eur J Radiol 81:2353-2357

14. Harvey N, Dennison E, Cooper C (2010) Osteoporosis: impact on health and economics. Nat Rev Rheumatol 6:99-105

15. Shen W, Chen J, Punyanitya M et al (2007) MRI-measured bone marrow adipose tissue is inversely related to DXA-measured bone mineral in Caucasian women. Osteoporos Int 18:641-647

16. Shen W, Scherzer R, Gantz M et al (2012) Relationship between MRImeasured bone marrow adipose tissue and hip and spine bone mineral density in African-American and Caucasian participants: the CARDIA study. J Clin Endocrinol Metab 97:1337-1346

17. Kanis JA (2002) Diagnosis of osteoporosis and assessment of fracture risk. Lancet 359:1929-1936

18. Kanis J, Borgstrom F, De Laet C et al (2005) Assessment of fracture risk. Osteoporos Int 16:581-589

19. Griffith JF, Yeung DK, Antonio GE et al (2005) Vertebral bone mineral density, marrow perfusion, and fat content in healthy men and men with osteoporosis: dynamic contrast-enhanced MR imaging and MR spectroscopy 1. Radiology 236:945-951

20. Griffith JF, Yeung DK, Antonio G et al (2006) Vertebral marrow fat content and diffusion and perfusion indexes in women with varying bone density: MR evaluation 1. Radiology 241:831-838

\section{Publisher's Note}

Springer Nature remains neutral with regard to jurisdictional claims in published maps and institutional affiliations.

\section{Author details}

'Department of Diagnostic Radiology, Faculty of Medicine, Suez Canal University, Ismailia, Egypt. ${ }^{2}$ Department of Neurosurgery, Faculty of Medicine, Suez Canal University, Ismailia, Egypt. 